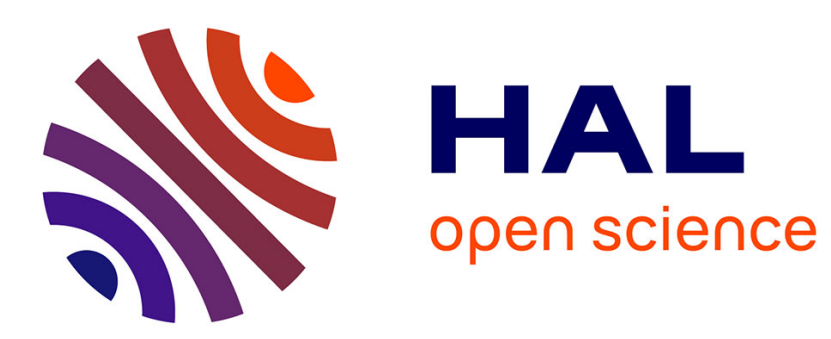

\title{
Correcting lower limb segment axis misalignment in gait analysis: A simple geometrical method
}

Alexandre Naaim, Alice Bonnefoy-Mazure, Stéphane Armand, Raphaël Dumas

\section{To cite this version:}

Alexandre Naaim, Alice Bonnefoy-Mazure, Stéphane Armand, Raphaël Dumas. Correcting lower limb segment axis misalignment in gait analysis: A simple geometrical method. Gait \& Posture, 2019, 72, pp. 34-39. 10.1016/j.gaitpost.2019.05.013 . hal-02264304

\section{HAL Id: hal-02264304 https://hal.science/hal-02264304}

Submitted on 6 Aug 2019

HAL is a multi-disciplinary open access archive for the deposit and dissemination of scientific research documents, whether they are published or not. The documents may come from teaching and research institutions in France or abroad, or from public or private research centers.
L'archive ouverte pluridisciplinaire HAL, est destinée au dépôt et à la diffusion de documents scientifiques de niveau recherche, publiés ou non, émanant des établissements d'enseignement et de recherche français ou étrangers, des laboratoires publics ou privés. 


\section{Accepted Manuscript}

Title: Correcting lower limb segment axis misalignment in gait analysis: a simple geometrical method

Authors: Alexandre Naaim (Investigation) (Formal analysis) (Methodology) (Software) (Writing - original draft) (Writing review and editing), Alice Bonnefoy-Mazure (Supervision) (Methodology) (Formal analysis) (Data curation) (Writing review and editing), Stéphane Armand (Conceptualization) (Supervision) (Writing - review and editing), Raphäl Dumas (Project administration) (Conceptualization) (Supervision) (Writing - review and editing)

PII:

DOI:

Reference: S0966-6362(18)31730-2

GAIPOS 6800

To appear in: $\quad$ Gait \& Posture

Received date: $\quad 18$ October 2018

Revised date: $\quad 30$ April 2019

Accepted date: $\quad 10$ May 2019

Please cite this article as: Naaim A, Bonnefoy-Mazure A, Armand S, Dumas R, Correcting lower limb segment axis misalignment in gait analysis: a simple geometrical method, Gait and amp; Posture (2019), https://doi.org/10.1016/j.gaitpost.2019.05.013

This is a PDF file of an unedited manuscript that has been accepted for publication. As a service to our customers we are providing this early version of the manuscript. The manuscript will undergo copyediting, typesetting, and review of the resulting proof before it is published in its final form. Please note that during the production process errors may be discovered which could affect the content, and all legal disclaimers that apply to the journal pertain. 


\section{Full paper}

Title: Correcting lower limb segment axis misalignment in gait analysis: a simple geometrical method

Alexandre Naaim ${ }^{\mathrm{a}}$, Alice Bonnefoy-Mazure ${ }^{\mathrm{b}}$, Stéphane Armand ${ }^{\mathrm{b}}$, Raphaël Dumas $^{\mathrm{a}}$

a Univ Lyon, Université Claude Bernard Lyon 1, IFSTTAR, LBMC UMR_T9406, F69622, Lyon, France

b Willy Taillard Laboratory of Kinesiology, Geneva University Hospitals and Geneva University, Switzerland

\section{Full adress :}

LBMC UMR_T9406

Laboratoire de Biomécanique et Mécanique des Chocs

Université Lyon1 - IFSTTAR

Batiment Oméga

43 Boulevard du 11 novembre 1918

69622 Villeurbanne Cedex, France

Willy Taillard Laboratory of Kinesiology

Geneva University Hospitals

4 Rue Gabrielle-Perret-Gentil

$\mathrm{CH}-1211$ Geneva 14, SWITZERLAND

\section{Authors e-mail:}

Alexandre Naaim : alexandre.naaim@univ-lyon1.fr

Alice Bonnefoy-Mazure: Alice.BonnefoyMazure@hcuge.ch

Stephane Armand: Stephane.Armand@hcuge.ch

Raphaël Dumas: raphael.dumas@ifsttar.fr

Corresponding author: alexandre.naaim@univ-lyon1.fr 


\section{Highlights}

- Hip rotation profile is prone to error due to segment axis misalignment

- Thigh medial-lateral axis is redefined a posteriori based on lower limb joint centres

- This geometrical method provides less knee crosstalk correction than reference methods

- This method provides equal hip rotation repeatability than reference methods

- This method is simpler than reference methods and is more widely applicable

\section{Abstract:}

Background: Obtaining precise and repeatable measurements is essential to clinical gait analysis. However, defining the thigh medial-lateral axis segment remains a challenge, with particular implications for the hip rotation profile. Thigh medial-lateral axis misalignment modifies the hip rotation profile and can result in a phenomenon called crosstalk, which increases knee adduction-abduction amplitude artificially.

Research question: This study proposes an a posteriori geometrical method based solely on segment anatomy that aims to correct the thigh medial-lateral axis definition and crosstalk-related error.

Methods: The proposed method considers the thigh medial-lateral axis as the normal to the mean sagittal plane of the lower limb defined by hip, knee and ankle joint centres during one gait cycle. Its performance was compared to that of an optimisation method which repositions the axis to reduce knee abduction- 
adduction variance. An existing dataset was used: 75 patients with a knee prosthesis undergoing gait analysis three months and one-year post-surgery.

Three-dimensional hip and knee angles were computed for two gait analysis sessions. Crosstalk was quantified using both the coefficient of determination $\left(r^{2}\right)$ between knee flexion-extension and adduction-abduction and the amplitude of knee adduction-abduction. The reproducibility of hip internal-external rotation was also quantified using the inter-trial, inter-session and inter-subject standard deviations and the intraclass coefficient (ICC).

Results: Crosstalk was significantly reduced from $r^{2}=0.67$ to $r^{2}=0.51$ by the geometrical method but remained significantly higher than with the optimisation method with a $r^{2}<0.01$.

Significances: Both methods allowed to improve the hip internal-external reproducibility from poor to moderate (original data: $I C C=0.34$, geometrical method: $I C C=0.65$, optimisation method $I C C=0.73)$. One advantage of the geometrical method is that, unlike the optimisation method, it does not require much movement, making it suitable for a wider range of patients.

Keywords: Knee crosstalk, Clinical gait analysis, Hip internal-external rotation Reproducibility, Gait 


\section{Introduction}

Clinical gait analysis helps clinicians identify gait impairments, thus guiding therapeutic choice (e.g. surgical planning, rehabilitation) in various pathologies (e.g. cerebral palsy, stroke) [1]. However, the joint kinematics involved are often computed with the Conventional Gait Model (CGM) [2] developed in the 80's, which has several shortcomings. The most common is marker mislocations, which can lead to segment axis misalignment; this can impact the accuracy of the hip rotation profile [3]. Hip rotation is a critical outcome, but one prone to error [4]. The medial-lateral axis direction is dependent on the positioning of a wand (or a medial marker) on the thigh. The wand should be positioned in the thigh's frontal plane. This plane can, however, be difficult to define in patients with skeletal deformities: for example, patients with cerebral palsy can exhibit major femoral growth abnormalities such as femoral torsion [5]. The resulting misalignment of this medial-lateral axis, which is used to define the flexion-extension axis of the distal joint, leads to a phenomenon called crosstalk. Crosstalk at the knee implies unrealistic adduction-abduction amplitude [3]. Furthermore, a misalignment of the medial-lateral axis can also lead to errors on the internal-external rotation of the joint [6].

Several experimental and computational solutions have been proposed to reduce these errors. One solution is to change the protocol (number and placement of markers) [7]. Devices such as the Knee Alignment Device can also be used to more accurately define the knee flexion-extension axis [8]. Alternatively, the knee flexion-extension axis can be defined using functional methods [9]. These methods are based on the joint kinematics rather than the segment anatomy and are applied to a specific movement maximising knee flexion. Functional methods can, however, be problematic for patients with possible adduction-abduction 
mobility or a limited flexion-extension range of motion. In addition, these methods are subject to soft tissue artefacts [10].

Another error-reducing solution might be to correct data retrospectively. The most classical a posteriori methods optimise the thigh coordinate system orientation to minimise the quadratic variance of the adduction-abduction angle [11] and possibly of the internal-external rotation angle [12]. Functional methods, too, can be applied retrospectively to gait records if the flexion during the swing phase is large enough [10,11]. Different methods have been used such as the axis transformation technics/SARA method [9] or STT methods [13]. Recent findings $[14,15]$ tends to suggest that optimisation methods like DynaKad [6] gave the best results. Multibody kinematics optimisation methods [16] can also define both the segment poses and the joint axes that best track marker trajectories. However, kinematics obtained from multibody kinematics optimisation methods tends to be strongly impacted by the kinematic model chosen. As a result, if the knee flexion-extension axis, which would correspond to the thigh medial-lateral axis direction, is poorly modelled the final results might be more reproducible but still inaccurate. In addition, all multibody kinematics optimisation methods are liable to suffer from overfitting.

Seeking to reduce crosstalk and enhance hip internal-external rotation reproducibility, the purpose of this study was to propose and evaluate an a posteriori geometrical method of correcting thigh medial-lateral orientation. Based on the position of the hip, knee and ankle joint centres, the method corrects segment axis misalignment so as to enhance the quality of both hip and knee kinematics measurements, in particular hip internal-external rotation and knee adduction-abduction. 


\section{Methods}

\section{Geometrical method}

Our geometrical method was inspired by an approach proposed for upper limb kinematics [17], where the upper arm medial-lateral axis is considered normal to the plane defined by three markers placed on the acromion process, humeral lateral epicondyle and ulnar styloid. To adapt this approach to the lower limb, we took the plane defined by the hip, knee and ankle joint centres.

Using the CGM, the thigh anterior-posterior axis was initially defined as that normal to the plane defined by the hip and knee joint centres and the wand marker position [2]. The medial-lateral axis of the thigh, which defines the knee flexion axis, lies on this normal plane and is orthogonal to the thigh superiorinferior axis defined from the knee joint centre to the hip joint centre. To correct these two axes, the sagittal plane of the lower limb was defined by the hip, knee and ankle joint centres. To avoid collinearity of these points and ensure that the normal was properly oriented to this sagittal plane, the average plane was calculated over all the instants of time when knee flexion was above $20^{\circ}$ during the movement. Then the thigh anterior-posterior axis was replaced by the cross product between the normal to the lower limb sagittal plane and the superiorinferior axis of the thigh. Finally, the medial-lateral thigh axis was replaced by another cross product between the newly defined thigh antero-posterior axis and the superior-inferior axis (Figure 1).

\section{Gait Analysis}

The method was applied retrospectively to a dataset from a cohort study of 118 patients undergoing total knee joint replacement surgery [18]. The initial project was approved by a local ethics committee (n CRE 09-307), and written consent was obtained from all participants. Each participant was subjected to gait 
analysis before, three months (M3) after and one year (M12) after the surgery. All acquisitions were performed at the Kinesiology Laboratory of Geneva University Hospitals using a Vicon 12-camera motion measurement system (VICON Mx3+; Vicon Peak, Oxford, UK). The cutaneous reflective markers were positioned according to the CGM [2] on the pelvis and the lower limb.

\section{Data Analysis}

To evaluate the performance of the proposed method on knee crosstalk and on hip kinematics reproducibility, only patients undergoing an $\mathrm{M} 3$ and $\mathrm{M} 12$ follow-up were considered. Although the true bone kinematics was unknown, it was assumed that after a total knee replacement knee adduction-abduction amplitude would be minimal. The hip internal-external rotation reproducibility was also one of our concern. After surgery, all patients went through a standard rehabilitation program for 5 to 6 weeks. As no further treatment was used after this 6 -weeks program, it was supposed that the hip internal-external rotation evolution would be minimal between M3 and M12 and could be then assessed.

Our geometrical method was compared to an optimisation method (DynaKad) proposed in the work of Baker et al. [6] which minimises knee adductionabduction during gait.

Data were analysed using MATLAB 2017a (The MathWorks, Natick, USA) and the Biomechanical Tool Kit [19]. The hip, knee and ankle joint centres computed using Nexus 1.8.5 (Vicon Peak, Oxford, UK), based on the CGM [2], were extracted from the C3D files. Finally, joint kinematics was computed using the 3D Kinematics and Inverse Dynamics toolbox proposed by Dumas and freely available on the MathWorks File Exchange [20]. This toolbox is based on the International Society of Biomechanics' recommendations for kinematics calculation [7]. With these recommendations, knee flexion is negative; however, it 
is common to define it as positive in clinical practice. For this reason, and for ease of reading, it was decided to inverse the knee flexion-extension in Figure 2. To obtain the original data as well as the corrected data yielded by the geometrical and optimisation methods, six quality assessments were performed on hip and knee kinematics. Knee crosstalk was assessed by analysing the amplitude of knee adduction-abduction and the coefficient of determination $\left(\mathrm{r}^{2}\right)$ between knee adduction-abduction and knee flexion-extension. Then, to assess the reproducibility of hip internal-external rotation, the inter-trial, inter-session and inter-subject standard deviations were investigated, in addition to the intersession-trial intraclass coefficient, following the statistical method proposed by Chia and Sangeux [21]. For the interpretation of the intraclass coefficient, the guidelines proposed by Koo and Lin [22] were used. These values need to be calculated on session with similar number of trials, a trial corresponding to a full gait cycle. In our data set, the trials' number was different for each session and for each subject. In order to take this difference into account, a bootstrap procedure was performed [23]. As the minimum of trial per session was seven, seven random trials were chosen for each session. The data analysis was then performed. This process has been done multiple times (1000) and the mean value for all the repetition used as the final value. The different parameters were calculated independently on each of 100 frames of the normalised gait cycle and the mean value with the $95 \%$ confidence interval given.

Finally, the distribution of the differences between M3 and M12 was plotted.

As all data were assumed to follow a normal distribution, a one-way repeated measure analysis of variance (ANOVA) was used to determine whether the resulting dataset (composed both of original data and of corrected data obtained with the geometrical and optimisation methods) had a significant effect on the 
above quality criteria. If a significant difference was found, a paired t-test was performed with significance level set at $p<0.01$. 


\section{Results}

Of the initial 118 patients in the dataset, 75 underwent M3 and M12 follow-ups (28 males and 47 females, Age: $68.4 \pm 7.1$ years old, BMl: $30.1 \pm 5.5 \mathrm{~kg} \cdot \mathrm{m}^{2}$ ) and were therefore included. These patients had at least 7 recorded gait cycles and a maximum knee flexion above $20^{\circ}$ in both sessions.

Mean and standard deviation (SD) of knee 3D kinematics are given for all conditions in Figure 2. The amplitude of knee adduction-abduction and the coefficient of determination between knee adduction-abduction and knee flexionextension are given in Table 1. Both correction methods had a significant effect on the coefficient of determination between knee adduction-abduction and knee flexion-extension, and on the amplitude of knee adduction-abduction. While the coefficient of determination between knee adduction-abduction and knee flexionextension was lower in the corrected data than in the original data, it nevertheless remains above 0.5 for the geometrical method. This result is confirmed by Figure 2, which shows increased knee adduction between $60 \%$ and $100 \%$ of the gait cycle. This part of the gait cycle corresponds to the swing phase during which maximum knee flexion occurs. Similarly, the amplitude of knee adduction-abduction was lower in the corrected data than in the original data. The lowest amplitude was obtained with the optimisation method $\left(6.5 \pm 0.2^{\circ}\right)$. The methods of correction had a significant effect on both the amplitude of knee adduction-abduction and the coefficient of determination between knee adduction-abduction and knee flexion-extension.

Regarding performance on hip internal-external rotation reproducibility, the intersession standard deviation was significantly lower when correction methods were used (Table 2). Both methods enhanced the inter-session reproducibility of hip internal-external rotation, with a lower standard deviation than in the original data 
(original: $8.7^{\circ}$, geometrical: $4.8^{\circ}$ and optimisation: $4.1^{\circ}$ ). The optimisation gave significant lower values than the geometrical method. Considering the ICC, both the geometrical and the optimisation improved the reproducibility of the original data from poor $(I C C=0.34)$ to a moderate reproducibility with a respective mean ICC of 0.64 and 0.72 (Table 2). The ICC obtained with the optimisation method was significantly greater than the geometrical method.

Figure 3 confirms this for the distribution of the differences between $\mathrm{M} 3$ and $\mathrm{M} 12$, with an error distribution centred around $0^{\circ}$ (geometrical: $-0.5^{\circ}$, optimisation: $\left.1.1^{\circ}\right)$

Both the geometrical and the optimisation methods had a positive significant effect on all the parameters evaluated. Knee adduction-abduction showed less crosstalk and hip internal-external rotation lower variability and inter-session difference. 


\section{Discussion}

The purpose of this study was to propose an a posteriori geometrical method based on the position of the hip, knee and ankle joint centres to correct the thigh medial-lateral axis. Our aim was to reduce crosstalk and improve hip internalexternal rotation reproducibility. Crosstalk was reduced; however, unlike the optimisation method proposed by Baker et al. [6] (Dynakad), our geometrical method did not completely eliminate it. The geometrical method improved the reproducibility of hip internal-external rotation between two sessions, like the optimisation method.

In this study, crosstalk has been chosen as an indicator of knee misorientation. Cross-talk is widely accepted as an indicator of misorientation of the thigh medial-lateral axis (considered as a surrogate of the knee flexion axis) $[6,11]$. For our population with knee total replacement, it was supposed to be a reasonable hypothesis as knee prostheses are generally designed to promote an asymptomatic knee kinematics pattern. Total knee prostheses do not provide a mechanism to allow varus/valgus movement at the knee. Therefore, in the population investigated in this study, the true knee varus-valgus range of motion was expected to be close to zero degree, and cross-talk was expected to be absent. However, Cross-talk is remains an indirect measurement of the supposed misorientation of the thigh medial-lateral axis and better gold standard exists for defining medio-lateral axis such as medical imaging. However, as gait analysis is always contaminated by the soft tissue artefact [24] (an artefact which is typically correlated to joint flexion-extension), even with a thigh medial-lateral axis matching at best the prosthesis flexion-extension axis, an apparent cross talk could be found. In this perspective, the optimisation method logically results in 
an apparent amplitude of adduction-abduction. However, this adductionabduction movement becomes totally uncorrelated to the flexion-extension.

We chose to compare our method to this optimisation method because it has been described as the best functional optimisation compared to EOS imaging [14]. This optimisation method was chosen also because it has been shown to perform better than marker set modification [11]. In addition, in a recent reproducibility study on the hip internal-external rotation for different functional methods [25], a similar optimisation method was found to be one with the highest reproducibility for hip internal-external rotation.

Both geometrical and optimisation methods were successful in reducing the knee crosstalk observed in our dataset. However, for both coefficient of determination and amplitude of adduction-abduction, the optimisation method provided better results, eliminating almost all the crosstalk with a correlation coefficient close to zero between knee flexion and adduction. This was expected, as the objective function to be minimised is the knee adduction-abduction amplitude. Consequently, the correlation between knee flexion-extension and adductionabduction was reduced by the optimisation process. In other words, the optimisation method, like functional methods [9-11], captures the flexionextension axis of the knee provided the flexion range of motion is sufficient. The geometrical method, while reducing crosstalk, did not fully eliminate it.

An important clinical parameter, hip internal-external rotation, unlike knee adduction-abduction, is commonly used for patient diagnosis or follow-up [4], so ensuring good reproducibility is essential [26]. Both the geometrical and optimisation methods tested here significantly improved the reproducibility of hip internal-external rotation relative to the original data. Considering the hip internalexternal rotation, the ICC found in our study were lower than the one obtained in a similar study [25]. They found an ICC of 0.88 for a similar optimisation method 
when an ICC of 0.73 was found in our study. This difference might be due to our two sessions being 9 months apart instead of being the same day as in this study [25]. It seems there is a larger variation than expected in a reproducibility study perform on the same day.

These results highlight one of our main limitation. It was supposed that the hip internal-external rotation evolution would be minimal during the 9 months between M3 and M12. However, as results suggest, it might be not true. Indeed, although no rehabilitation program was performed after $\mathrm{M} 3$, potential modification of gait pattern could probably occur. This can be explained by the fact that patients with knee arthrosis can have high pain level before surgery resulting in low level of activity or walking. As a result, if the surgery is successful, patient's activity level and walking distance can increase. This could allow to strengthen abductor muscles which might take more than the 3-month rehabilitation program to fully recover. As the gluteus and the tensor of fascia lata, which are the main hip abductor muscles, are also hip rotator [27] modification of the hip internalexternal rotation profile could then have been expected between M3 and M12. As a result, the lower ICC obtained in our study might be mainly due to intrinsic subject variability rather than a poorer performance of our method. We might expect better result with our geometrical method if we performed a reproducibility study with sessions during the same day or during the same week.

As the main correction of hip internal-external rotation consists in adding an offset in both methods, it is interesting to look at hip data obtained from the true bone kinematics. Using bi-plane fluoroscopy on subjects without total knee replacement, Fiorentino et al. [28] found that the hip internal-external rotation was comprised between $-15^{\circ}$ and $0^{\circ}$ during gait and that the soft tissue artefact tended to shift it towards internal rotation. These values are similar both to those from our original dataset and to those obtained after correction by the 
geometrical method. Conversely, the optimisation method seems to result in hip internal-external rotation being more shifted toward external rotation.

From a clinical point of view, the geometrical method has some advantages over the optimisation method. As the method is based on segment geometry rather than joint kinematics and does not include any minimisation process, it should be less sensitive to soft tissue artefacts and free from overfitting issues. In addition, it does not require any movement other than a slight knee flexion. This makes it particularly appropriate in certain contexts, for instance for patients with crouch gait. However, a knee flexion of at least $20^{\circ}$ must be reach by the patient to be able to use the geometrical method. As a result, it might not be adapted for patients with stiff-knee gait. This limitation is also valid for other knee cross-talk correction methods, like functional or optimisation methods, as they are mainly based on knee movements.

One of the main advantages of this method is also its main weakness. As the geometrical method is mainly based on segment anatomy, skeletal deformities may affect the results. Pathologies commonly analysed in a gait laboratory, such as cerebral palsy, can involve torsional deformities that could compromise the results of the geometrical method. However, where the deformity remains the same across the different sessions, hip internal-external rotation reproducibility should remain constant even if the crosstalk correction is affected. Moreover, as the geometrical method is exclusively based on joint centre location, it might be sensitive to errors on joint centres, as has been reported for the hip [7,29]. However, considering the large distance between the hip, the knee and the ankle, it can be assumed that any effect on the definition of the sagittal plane of the lower limb would be minimal. To conclude on this, however, would have required testing the method on patients with skeletal deformities. 


\section{Conclusion}

The geometrical method proposed here to correct misalignment of the thigh medial-lateral axis performs similarly to an optimisation method in improving hip internal-external rotation reproducibility, but not in fully eliminating knee crosstalk. The main advantage of the geometrical method includes the fact that it is based on simple segment geometry, while the optimisation method is based on a more complex minimisation process. Moreover, if the main objective of a posteriori correction of gait data is to obtain repeatable hip internal-external rotation, the geometrical method may be an alternative choice since it is more widely applicable, including in situations where the patient's range of motion is limited. However, if the main objective is to correct crosstalk, optimisation methods should be chosen provided there is a sufficient range of motion.

\section{Conflict of Interest Statement}

The authors certify that there is no conflict of interest or any financial and personal relationships with other people or organisations that could inappropriately influence this work

Acknowledgements: We thank Katia TURCOT and Yoshimasa SAGAWA JUNIOR for their contributions to data acquisition.

\section{References :}

[1] T.A.L. Wren, N.Y. Otsuka, R.E. Bowen, A.A. Scaduto, L.S. Chan, M. Sheng, R. Hara, R.M. Kay, Influence of gait analysis on decision-making for lower extremity orthopaedic surgery: Baseline data from a randomized controlled trial, Gait Posture. $34 \quad$ (2011) 364-369. 
doi:10.1016/J.GAITPOST.2011.06.002.

[2] R. Davis, S. Ounpuu, D. Tyburski, J.J. Gage, I. Davis, S. Ounpuu, D. Tyburski, J.J. Gage, A gait analysis data collection and reduction technique, Hum. Mov. Sci. 10 (1991) 575-587. doi:10.1016/01679457(91)90046-z.

[3] U. Della Croce, A. Leardini, L. Chiari, A. Cappozzo, Human movement analysis using stereophotogrammetry. Part 4: assessment of anatomical landmark misplacement and its effects on joint kinematics., Gait Posture. 21 (2005) 226-37. doi:10.1016/j.gaitpost.2004.05.003.

[4] T.A.L. Wren, C. Lening, S.A. Rethlefsen, R.M. Kay, Impact of gait analysis on correction of excessive hip internal rotation in ambulatory children with cerebral palsy: a randomized controlled trial, Dev. Med. Child Neurol. 55 (2013) 919-925. doi:10.1111/dmcn.12184.

[5] S. Gose, T. Sakai, T. Shibata, T. Murase, H. Yoshikawa, K. Sugamoto, Morphometric analysis of the femur in cerebral palsy: 3-dimensional CT study, J. Pediatr. Orthop. $30 \quad$ (2010) 568-574. doi:10.1097/BPO.0b013e3181e4f38d.

[6] R. Baker, L. Finney, J. Orr, A new approach to determine the hip rotations profile from clinical gait analysis data, Hum. Mov. Sci. 18 (1999) 655-667. doi:10.1016/S0167-9457(99)00027-5.

[7] G. Wu, S. Siegler, P. Allard, C. Kirtley, A. Leardini, D. Rosenbaum, M. Whittle, D.D. D’Lima, L. Cristofolini, H. Witte, O. Schmid, I. Stokes, D.D. D'Lima, L. Cristofolini, H. Witte, O. Schmid, I. Stokes, ISB recommendation on definitions of joint coordinate system of various joints for the reporting of human joint motion--part I: ankle, hip, and spine, J. Biomech. 35 (2002) 543-548. doi:10.1016/S0021-9290(01)00222-6.

[8] R.W. Baker, Measuring Walking: A Handbook of Clinical Gait Analysis, 
2014.

[9] R.M. Ehrig, W.R. Taylor, G.N. Duda, M.O. Heller, A survey of formal methods for determining functional joint axes., J. Biomech. 40 (2007) 2150-7. doi:10.1016/j.jbiomech.2006.10.026.

[10] M. Sangeux, A. Barré, K. Aminian, Evaluation of knee functional calibration with and without the effect of soft tissue artefact, J. Biomech. 62 (2017) 53-59. doi:10.1016/j.jbiomech.2016.10.049.

[11] A.G. Schache, R. Baker, L.W. Lamoreux, Defining the knee joint flexionextension axis for purposes of quantitative gait analysis: an evaluation of methods., Gait $\quad$ Posture. $24 \quad$ (2006) 100-109. doi:10.1016/j.gaitpost.2005.08.002.

[12] L.-P. Rivest, A correction for axis misalignment in the joint angle curves representing knee movement in gait analysis., J. Biomech. 38 (2005) 1604-1611. doi:10.1016/j.jbiomech.2004.07.031.

[13] M.H. Schwartz, A. Rozumalski, A new method for estimating joint parameters from motion data., J. Biomech. 38 (2005) 107-16. doi:10.1016/j.jbiomech.2004.03.009.

[14] C. Sauret, H. Pillet, W. Skalli, M. Sangeux, On the use of knee functional calibration to determine the medio-lateral axis of the femur in gait analysis: Comparison with EOS biplanar radiographs as reference, Gait Posture. 50 (2016) 180-184. doi:10.1016/j.gaitpost.2016.09.008.

[15] E. Passmore, H.K. Graham, M. Sangeux, Defining the medial-lateral axis of the femur: Medical imaging, conventional and functional calibration methods lead to differences in hip rotation kinematics for children with torsional deformities, J. Biomech. 69 (2018) 156-163. doi:10.1016/J.JBIOMECH.2018.01.018.

[16] M. Begon, M.S. Andersen, R. Dumas, Multibody Kinematics Optimization 
for the Estimation of Upper and Lower Limb Human Joint Kinematics: A Systematized Methodological Review, J. Biomech. Eng. 140 (2018) 030801. doi:10.1115/1.4038741.

[17] R. a Cooper, M.L. Boninger, S.D. Shimada, B.M. Lawrence, Glenohumeral joint kinematics and kinetics for three coordinate system representations during wheelchair propulsion., Am. J. Phys. Med. Rehabil. 78 (1999) 435446. doi:10.1097/00002060-199909000-00006.

[18] A. Bonnefoy-Mazure, S. Armand, Y. Sagawa, D. Suvà, H. Miozzari, K. Turcot, Knee Kinematic and Clinical Outcomes Evolution Before, 3 Months, and 1 Year After Total Knee Arthroplasty, J. Arthroplasty. 32 (2017) 793-800. doi:10.1016/j.arth.2016.03.050.

[19] A. Barre, S. Armand, Biomechanical ToolKit: Open-source framework to visualize and process biomechanical data, Comput. Methods Programs Biomed. 114 (2014) 80-87. doi:10.1016/j.cmpb.2014.01.012.

[20] R. Dumas, 3D Kinematics and Inverse Dynamics, (2016). https://fr.mathworks.com/matlabcentral/fileexchange/58021-3d-kinematicsand-inverse-dynamics (accessed June 6, 2018).

[21] K. Chia, M. Sangeux, Quantifying sources of variability in gait analysis, Gait Posture. 56 (2017) 68-75. doi:10.1016/J.GAITPOST.2017.04.040.

[22] T.K. Koo, M.Y. Li, A Guideline of Selecting and Reporting Intraclass Correlation Coefficients for Reliability Research., J. Chiropr. Med. 15 (2016) 155-63. doi:10.1016/j.jcm.2016.02.012.

[23] M.K. Campbell, D.J. Torgerson, Bootstrapping: estimating confidence intervals for cost-effectiveness ratios, QJM. 92 (1999) 177-182. doi:10.1093/qjmed/92.3.177.

[24] V. Camomilla, R. Dumas, A. Capozzo, Human movement analysis: The soft tissue artefact issue, J. Biomech. 62 (2017) 1-4. 
doi:10.1016/J.JBIOMECH.2017.09.001.

[25] M. Sangeux, Computation of hip rotation kinematics retrospectively using functional knee calibration during gait, Gait Posture. 63 (2018) 171-176. doi:10.1016/j.gaitpost.2018.05.011.

[26] R.A. Brand, R.D. Crowninshield, Comment on criteria for patient evaluation tools., J. Biomech. 14 (1981) 655. doi:10.1016/0021-9290(81)90093-2.

[27] S.J. Preece, P. Graham-Smith, C.J. Nester, D. Howard, H. Hermens, L. Herrington, P. Bowker, The influence of gluteus maximus on transverse plane tibial rotation, Gait Posture. 27 (2008) 616-621. doi:10.1016/j.gaitpost.2007.08.007.

[28] N.M. Fiorentino, P.R. Atkins, M.J. Kutschke, J.M. Goebel, K.B. Foreman, A.E. Anderson, Soft tissue artifact causes significant errors in the calculation of joint angles and range of motion at the hip, Gait Posture. 55 (2017) 184-190. doi:10.1016/j.gaitpost.2017.03.033.

[29] H. Kainz, C.P. Carty, D.G. Lloyd, L. Modenese, S. Maine, H.P.J. Walsh, Effects of hip joint centre mislocation on gait kinematics of children with cerebral palsy calculated using patient-specific direct and inverse kinematic models, Gait Posture. $57 \quad$ (2017) 154-160. doi:10.1016/j.gaitpost.2017.06.002. 


\section{Figures:}

Figure 1:

\section{Plane definition}

\section{Axis reorientation}
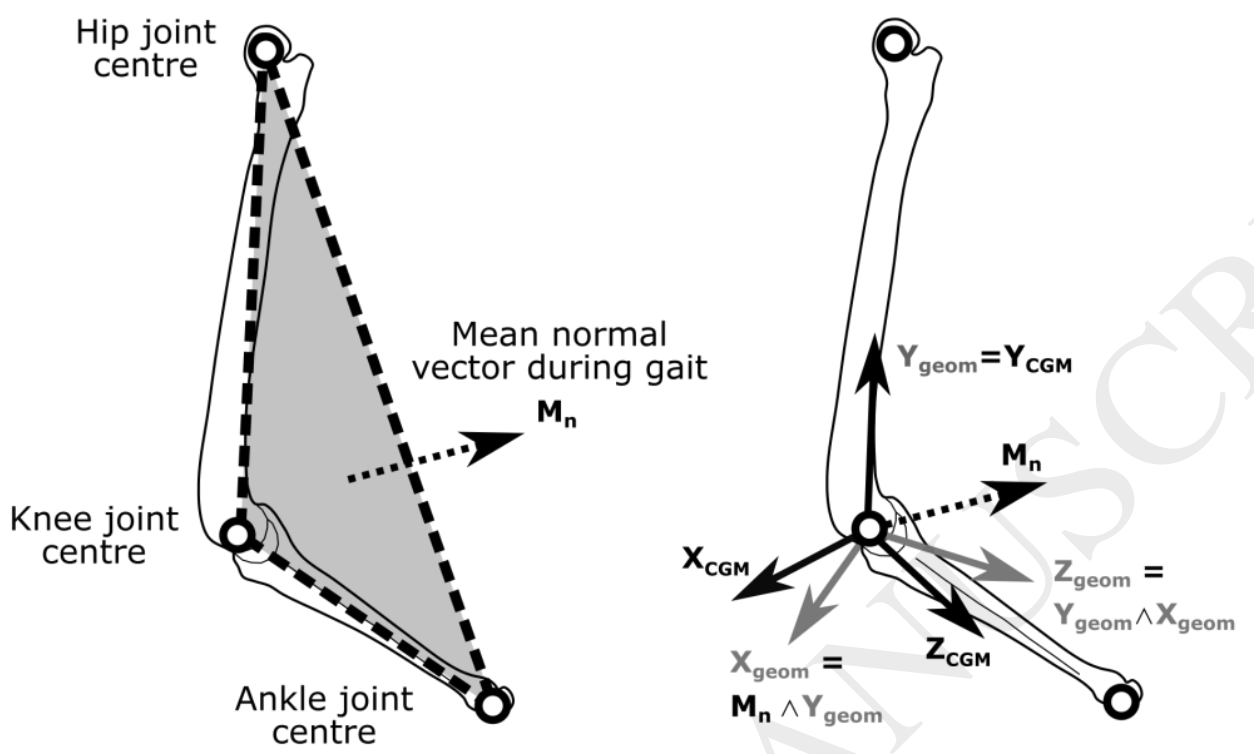

Figure 2:
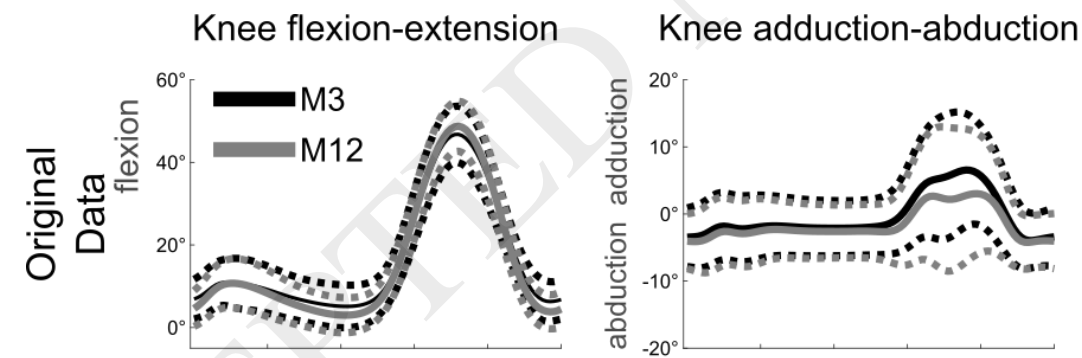

Hip internal-external rotation
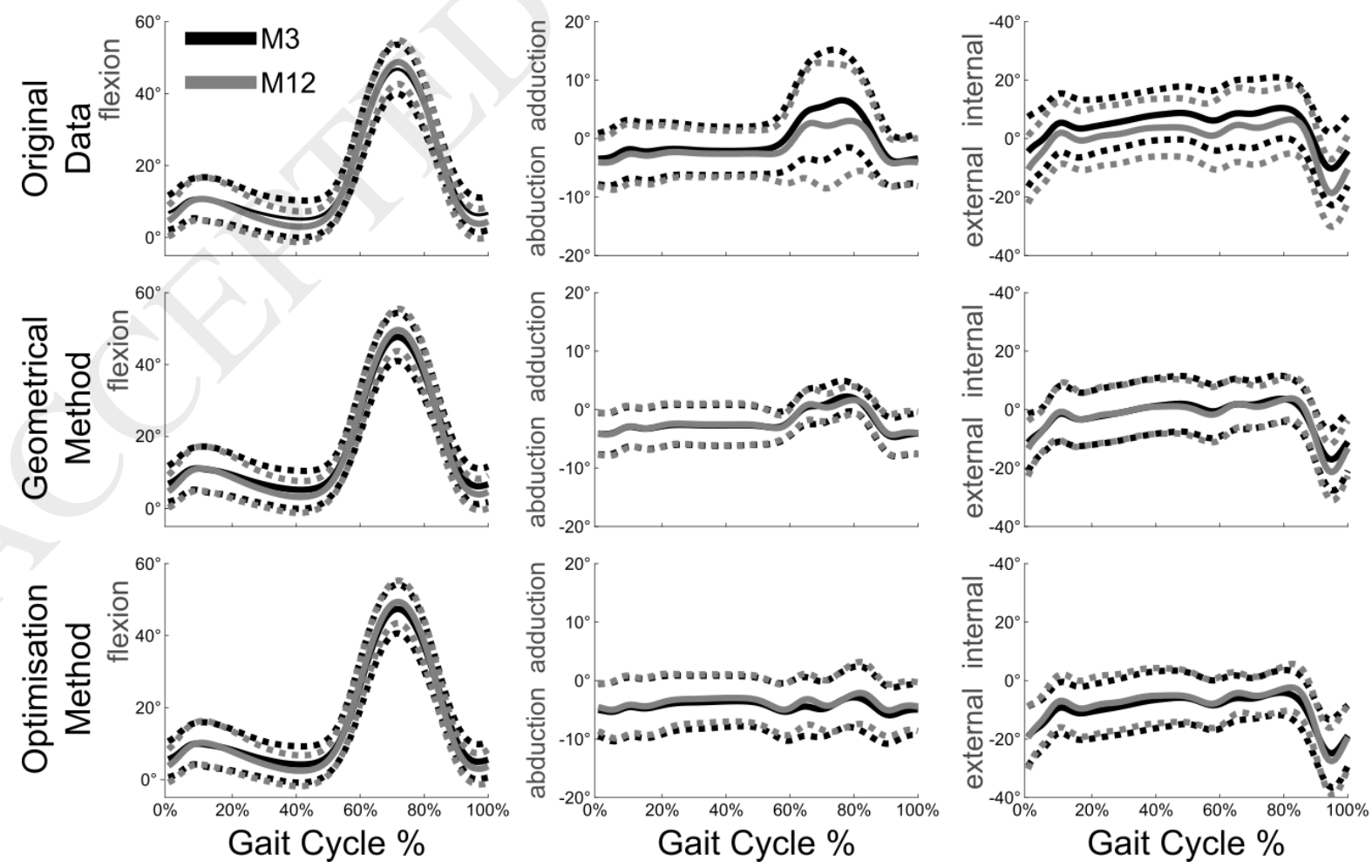
Figure 3:

Hip internal-external rotation

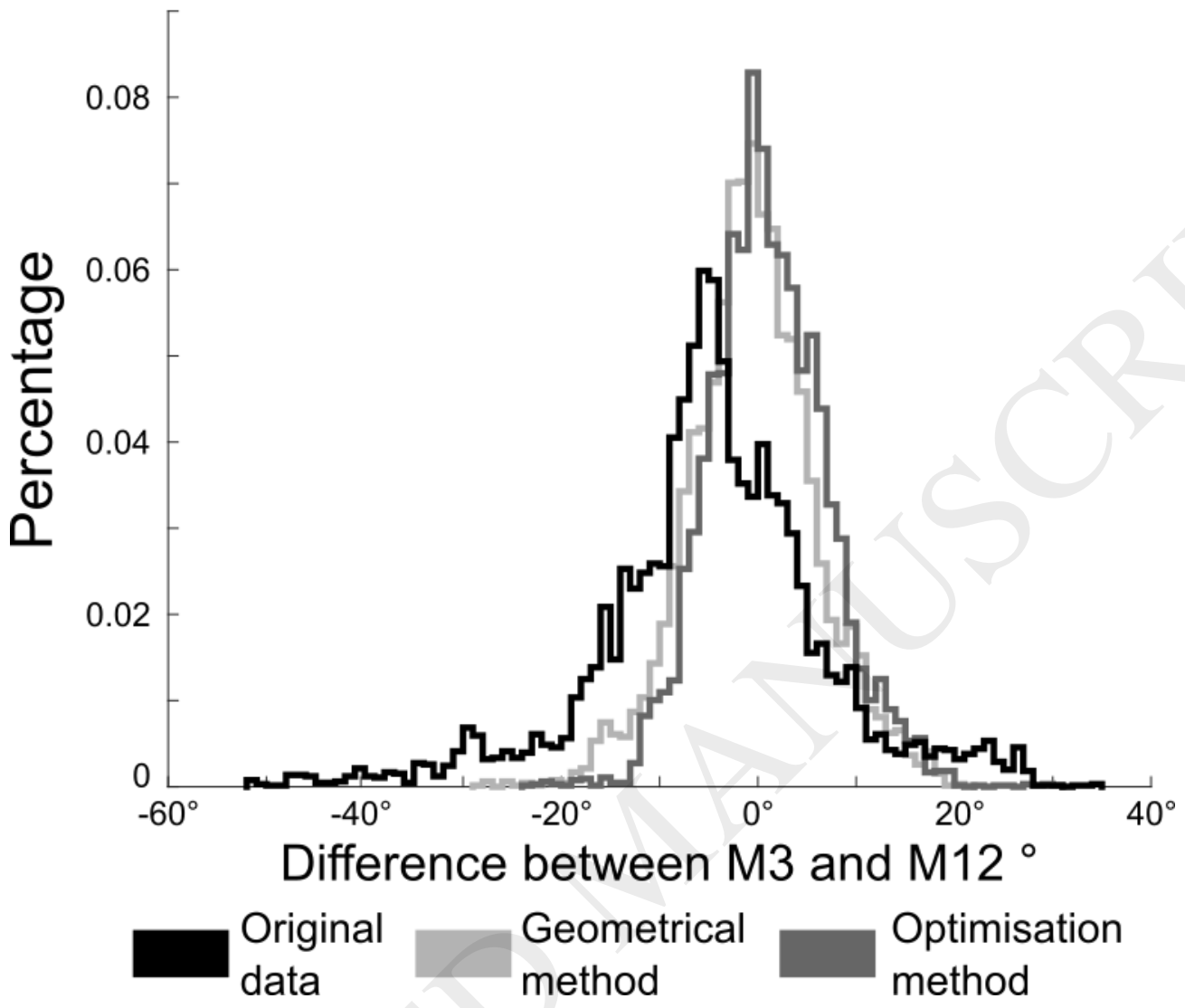

\section{Figure captions:}

\section{Figure 1:}

Schematics of the construction of the thigh frame using the geometrical method

\section{Figure 2:}

Mean (solid) with \pm 1 -standard deviation (dotted) of hip internal-external rotation, knee flexion-extension and adduction-abduction for gait analysis three months 
(M3) and one year (M12) after surgery: original data and data corrected using geometrical and optimisation methods.

\section{Figure 3:}

Normalised histogram of the difference distribution between gait analysis three months (M3) and one year (M12) after surgery, for hip internal-external rotation for original data and both correction methods. 


\section{Tables:}

Table 1: Effect of the correction methods on knee crosstalk

\begin{tabular}{lccc}
\hline & $\begin{array}{c}\text { Original } \\
\text { data } \\
\text { Mean }(95 \mathrm{Cl} \%)\end{array}$ & $\begin{array}{c}\text { Geometrical } \\
\text { method } \\
\text { Mean }(95 \mathrm{Cl} \%)\end{array}$ & $\begin{array}{c}\text { Optimisation } \\
\text { method } \\
\text { Mean }(95 \mathrm{Cl} \%)\end{array}$ \\
\begin{tabular}{l} 
Crosstalk evaluation \\
\hline $\mathrm{r}^{2}$ knee (flexion-
\end{tabular} & $0.67(0.61-0.72)$ & $0.51(0.45-0.56)$ & $0.01>$ *\# \\
$\begin{array}{l}\text { adduction) } \\
\text { Amplitude knee AA }\left(^{\circ}\right)\end{array}$ & $13.5^{\circ}(12.4-14.5)$ & $9.9^{\circ}(9.1-10.6)$ & $6.5^{\circ}(6.1-7.0)$
\end{tabular}

Table 2: Effect of the correction methods on hip internal-external rotation reproducibility.

\begin{tabular}{lccc}
\hline Variance & $\begin{array}{c}\text { Original } \\
\text { data } \\
\text { Mean }(95 \mathrm{Cl} \%)\end{array}$ & $\begin{array}{c}\text { Geometrical } \\
\text { method } \\
\text { Mean }(95 \mathrm{Cl} \%)\end{array}$ & $\begin{array}{c}\text { Optimisation } \\
\text { method } \\
\text { Mean }(95 \mathrm{Cl} \%)\end{array}$ \\
\hline Inter-subject & $6.7^{\circ}\left(6.4^{\circ}-6.9^{\circ}\right)$ & $7.8^{\circ}\left(7.7^{\circ}-8.0^{\circ}\right)^{\star}$ & $8.3^{\circ}\left(8.1^{\circ}-8.4^{\circ}\right)^{\star \#}$ \\
Inter-session & $8.7^{\circ}\left(8.6^{\circ}-8.8^{\circ}\right)$ & $4.8^{\circ}\left(4.7^{\circ}-4.9^{\circ}\right)^{\star}$ & $4.1^{\circ}\left(3.9^{\circ}-4.3^{\circ}\right)^{\star \#}$ \\
Inter-trial & $2.8^{\circ}\left(2.7^{\circ}-2.9^{\circ}\right)$ & $2.9^{\circ}\left(2.8^{\circ}-3.0^{\circ}\right)$ & $2.9^{\circ}\left(2.8^{\circ}-3.1^{\circ}\right)$ \\
ICC & & & \\
\hline ICC & $0.34(0.33-0.35)$ & $0.65(0.64-0.66)^{*}$ & $0.73(0.71-0.74)$ \\
$\star \#$
\end{tabular}

Cl: Confidence interval, ICC : Intra Class Correlation * vs Original $p<0.01$, \# vs Geometrical $p<0.01$ 